
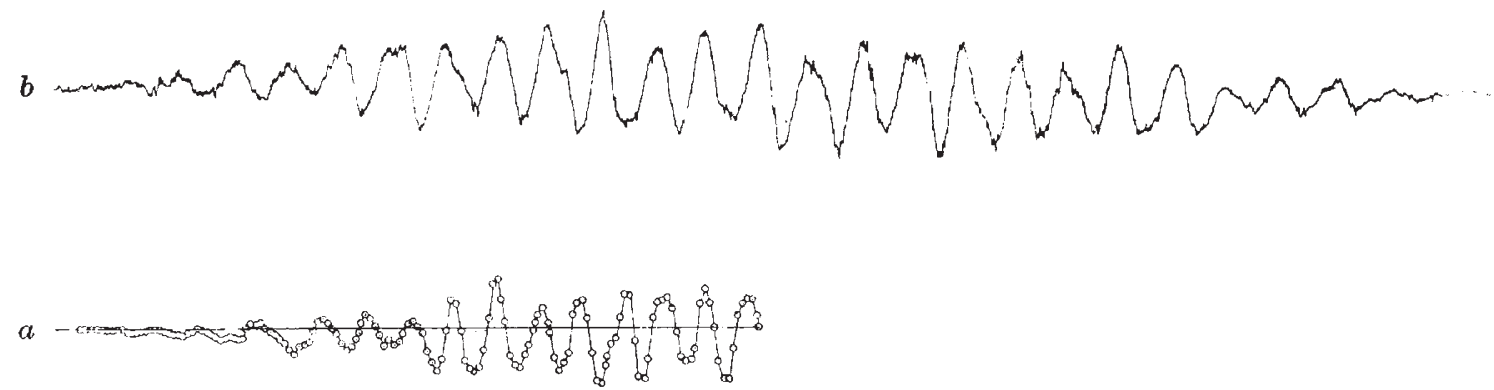

Fig. 1. Transient ESR spectra of benzophenone radical anion in a highly alkaline isopropanol solution $10 \mathrm{mis}$ after its formation. The lower trace $(a)$ was obtained by the stepped field method with time resolution and the upper trace $(b)$ by the continuous sweep method and recording at

cessing, and we recommend its use for radical identification in simple cases. Our method gives immediate kinetic data, and after traversing the spectrum we have information on 480 different ESR spectra as a function of time. In our method the presence of a secondary radical produced by reaction of the initial species would easily and immodi. ately be detected because we have the spectra of the radicals in the system over their entire lifetimes. The other technique does not observe the system continuously. Furthermore, our method is capable of providing exact measurements of line positions by its ability to locate the crossover points of the derivative curves.

We thank the Gas Council for a maintenance grant to A. F. S., and the Science Research Council for financial support.

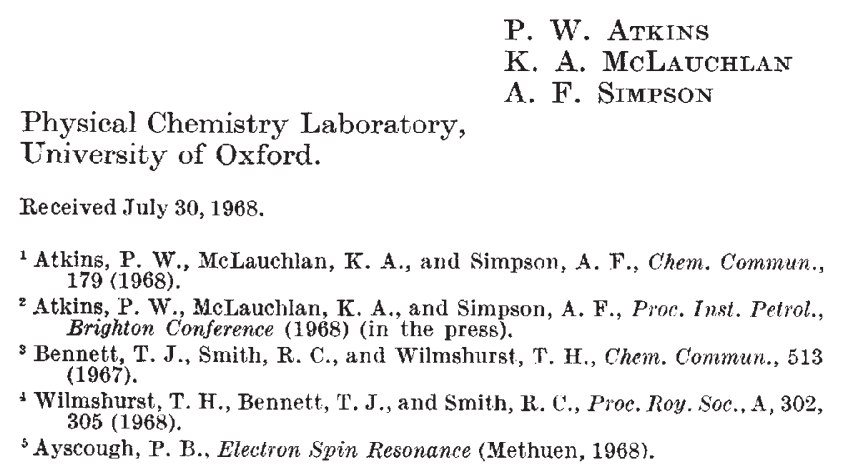

\section{Determination of Purity by Nuclear Magnetic Resonance}

Burnett and Muller' recently reported a method of measuring melting points using nuclear magnetic resonance spectroscopy. For the past 18 months we have been using a nuclear magnetic resonance technique to determine the purity of organic compounds.

Our experimental procedure is somewhat simpler than that reported ${ }^{1}$. A high-resolution spectrometer (Associated Electrical Industries type $R S 2$ ) oporating at 40 $\mathrm{MHz}$ records the proton spectrum of the material investigated from about $20^{\circ}$ below the melting point up to the temperature at which the sample is completely molten. A non-spinning sample tube is used, so that the magnetic field inhomogeneity over the sample volume of $2 \times 10^{-7} \mathrm{~m}^{3}$ $\left(0 \cdot 2 \mathrm{~cm}^{3}\right)$ is about $30 \mathrm{~Hz}$, and this determines the width of the line from the molten liquid. The width of the line from the solid material is typically of the order of $10^{4} \mathrm{~Hz}$, so that the magnetic field sweep can easily be adjusted to show only the line from the liquid. The intensity of the liquid line is measured from the areat under the peak by cutting out and weighing peaks, and the fraction melted is thus found. A graph of the tempera. ture of measurement against the reciprocal of the fraction melted can be plotted. This is linear for systems not forming solid solutions, the slope giving the purity of the sample and the intercept on the temperature axis the melting point of the absolutely pure material.

This plot is linear for various samples of phenol with water added as impurity, and the purity so found agrees well with that expected for samples botween 94 and $99 \cdot 6$ mole per cent purity. Mixtures of phenol and $o, m$ or $p$-cresol containing not less than 90 per cont phenol behave in an interesting way. While the phenol- $p$ cresol system gives a linear plot of temperature against the reciprocal of the fraction melted, mixtures of phenol with $a$ or $m$-cresol give curves, showing the existence of solid solutions. Values of the distribution coefficient of the impurity in the solid and liquid phases can be derived from the nuclear magnetic resonance data. Such values are important-for example, in deciding whether zone refining is a feasible method for the purification of a compound.

We have also studied mixtures of 1 and 2-methylnaphthalenes over the entire concentration range. Measurements of the intensity of the liquid signal again give the distribution coefficient in the solid and liquid, and also enable the phase diagram of this system to be drawn much more accurately than previously ${ }^{2}$. This useful technique should find wide application in the study of solid-liquid phase diagrams.

As a method of purity control, nuclear magnetic resonance is quicker than other cryoscopic methods and, because a fraction melted of 1 per cent can be readily measured, the requirements for very accurate temperature control are not stringent. Samples covering a wide range of purity can be studied.

Experiments in progress using a simple commercial nuclear resonance apparatus should enable the extension of the method to industrial purity control.

\section{E. F. G. Herington}

Division of Chemical Standards,

\section{J. LAWRENSON}

National Physical Laboratory,

Teddington, Middlesex.

Received July 17, 1968.

${ }^{1}$ Burnett, L. J., and Muller, B. H., Nature, 219, 59 (1968).

${ }^{2}$ Coulson, E. A., J. Soc. Chem. Indust., 60, 123 (1941).

\section{Analogy between Vortex Stretching by Drag-reducing Additives and Vortex Stretching by Fine Suspensions}

THE reduction of drag in water and other solvents by the addition of traces of certain long-chain molecules is a well known effect, which Gadd ${ }^{1}$ explains by a vortex stretching mechanism. He discusses the transient diffu- 